\title{
$\alpha$-enolase and caveolin alterations in the heart of rats having undergone gentamicin-induced toxicity
}

\author{
QUAN-YONG HUANG ${ }^{1}$, XUE-FANG LI $^{2}$ and SHUI-PING LIU ${ }^{3}$ \\ ${ }^{1}$ Department of Pathology, School of Basic Medical Sciences, Dali University; ${ }^{2}$ School of Basic Medical Sciences, \\ Dali University, Dali, Yunnan 671000; ${ }^{3}$ Department of Forensic Pathology, \\ Zhongshan School of Medicine, Sun Yat-Sen University, Guangzhou 510080, P.R. China
}

Received October 10, 2011; Accepted December 16, 2011

DOI: $10.3892 / \mathrm{mmr} .2011 .728$

\begin{abstract}
Gentamicin (GM)-induced heart damage is associated with alterations in expression levels of various enzymes and proteins. The aim of the present study was to investigate GM-induced alterations in cardiac $\alpha$-enolase and caveolin after GM administration. Male Wistar rats were randomly divided into two groups: a control group and a GM group. The GM group intraperitoneally received GM at a single dose of $7 \mathrm{mg} / \mathrm{kg}$ for 8 days, while the controls were given the same amount of saline via the same route. On Day 9, the rats were anesthetized and a thoracotomy was performed in all animals. Immunohistochemical analysis was performed to evaluate protein expression of $\alpha$-enolase and caveolin. Sections were analyzed by digital image analysis. Our results revealed that cardiac protein expression of $\alpha$-enolase and caveolin was altered after GM-induced toxicity in the rat. The expression of $\alpha$-enolase and caveolin was significantly increased after GM-induced toxicity, as determined by integrated optical density analysis, when compared with the control $(\mathrm{P}<0.05)$. The current findings indicate that such changes in protein expression may be reflected in abnormal cardiac function, and the proteins identified in this study may be useful for elucidating the mechanisms underlying GM-induced toxicity and may also provide various clues for further investigations.
\end{abstract}

\section{Introduction}

Gentamicin (GM) is considered to be an important antibiotic against life-threatening, severe gram-negative bacterial infections (1), yet it also exhibits severe side effects such as nephrotoxicity $(2)$ and inner ear injury $(3,4)$. Nephrotoxicity is

Correspondence to: Dr Quan-Yong Huang, Department of Pathology, School of Basic Medical Sciences, Dali University, Xiaguan Wanhua Road, Dali, Yunnan 671000, P.R. China

E-mail: hqy0726@126.com

Key words: $\alpha$-enolase, caveolin, gentamicin-induced toxicity the main side effect of GM (5). Thus, its clinical use is partially limited due to its nephrotoxic effect (6).

Tested hypotheses concerning the mechanisms involved in GM-induced nephrotoxicity are as follows. Evidence indicates that free radicals are important mediators of GM nephrotoxicity $(7,8)$. It has been shown that GM-induced renal dysfunction was attributable to enhanced oxidative stress (9), as revealed by decreased superoxide dismutase and catalase activities, glutathione depletion and increased lipid peroxidation (8). In addition, it has been suggested that the c-Jun $\mathrm{N}$-terminal kinase (JNK) signaling pathway may be involved in the secondary apoptosis of spiral ganglion cells (SGCs) induced by intracochlear GM injection (9), and the formation of aminoglycoside-iron complexes have been proposed to be the major mechanisms in the development of GM-induced acute renal failure (ARF) (10). Moreover, it has been demonstrated that GM-induced nephrotoxicity leads to increased urinary losses of carnitine, and carnitine deficiency should be viewed as a mechanism during the development of GM-induced ARF (11). GM-induced nephrotoxicity has also been associated with marked upregulation of Kim-1, TIMP1 (12) and mitogen-activated protein kinase (MAPK) (2), but downregulation of MMP-9 (13).

Extended use or excessive dosages of GM have been found to result in irreversible damage to the inner ear such as inner ear hair cell loss and sensorineural hearing loss. Apoptotic cell death is considered to play a key role in this injury $(3,4)$.

It has been shown that the GM-induced toxicity is involved in changes in superoxide dismutase and catalase activities, glutathione and lipid peroxidation (8) and respiratory components (cytochrome c, NADH) (14). However, little is known concerning the changes in $\alpha$-enolase and caveolin expression in the heart having undergone GM-induced toxicity.

The present study used immunohistochemistry to evaluate the expression of $\alpha$-enolase and caveolin in the heart, and determine whether the protein expression is altered upon GM-induced toxicity.

\section{Materials and methods}

Sixteen healthy adult male Wistar rats, weighing 210-250 g, were used in this study. All animals were housed individually in stainless-steel wire-bottomed cages in an air-conditioned 
room at $22^{\circ} \mathrm{C}$ with $50 \%$ relative humidity and a 12-h light/dark cycle. Rats were fed with a standard pellet chow and water throughout the experimental period. All procedures described in this study were approved by the Ethics Committee of Sun Yat-Sen University.

Animals and study design. Animals were divided randomly into 2 groups, each with 8 rats. GM group animals were induced by an intraperitoneal injection of GM at a dose of $100 \mathrm{mg} / \mathrm{kg} /$ day for 8 days, and the control group animals were treated with normal saline for 8 days in equal volumes as for the experimental groups $(7,15)$.

After 8 days of treatment with GM (100 mg/kg/day), control and experimental animals were anesthetized with xylazine $(10 \mathrm{mg} / \mathrm{kg})$ and ketamine $\mathrm{HCl}(50 \mathrm{mg} / \mathrm{kg})$ and sacrificed. A thoracotomy was then performed. The heart was harvested, fixed in phosphate-buffered $10 \%$ formalin, embedded in paraffin wax, and sectioned $(4 \mu \mathrm{m})$ for light microscopy and immunohistochemical examination.

Histopathologic examinations. Heart specimens from each group were removed for histopathological evaluation. The heart tissues were fixed in $10 \%$ formalin, embedded in parafin, sectioned at $4 \mu \mathrm{m}$, and then stained with hematoxylin and eosin. The slides were coded and semi-quantitative analysis of the sections was performed without knowledge of the treatment protocol. Pathologic changes were evaluated in these tissues $(16,17)$.

Tissue sections and immunohistochemical staining. All rat hearts were immersed in $4 \%$ formaldehyde buffered with phosphate-buffered saline (PBS, pH 7.2), before being embedded in paraffin and sectioned coronally with a microtome into $4-\mu \mathrm{m}$ sections. After deparaffinization, all sections were immersed in $0.3 \% \mathrm{H}_{2} \mathrm{O}_{2}$-PBS for $10 \mathrm{~min}$ and incubated with PBS containing $1 \%$ normal goat serum to reduce non-specific reactions. After incubation with either rabbit anti$\alpha$-enolase, or anti-caveolin (Santa Cruz Biotechnology, USA) overnight at $4^{\circ} \mathrm{C}$, tissue sections were washed three times in phosphate-buffered saline (PBS) and incubated with biotinconjugated secondary antibody for $1 \mathrm{~h}$ at room temperature according to the manufacturer's instructions. The specimens were then incubated with peroxidase-conjugated avidin-biotin for $30 \mathrm{~min}$ at room temperature. After washing, the sections were incubated with diaminobenzidine (DAB) as chromogen, and counterstained with hematoxylin (18-20). PBS was substituted for the primary antibody as the negative control.

Integrated optical density (IOD), a parameter representing expression levels of $\alpha$-enolase and caveolin in cardiac tissue, was determined using a cast-grid microscope (MetaMorph/ DP10/Bx41, UIC/Olympus, US/JP) together with an imageanalysis program (MetaMorph offline, version 4.65). Under a magnification of $x 400$, five images were captured in each immunostained section and the average was calculated $(19,20)$.

Statistical analyses. Statistical analyses were performed by using SPSS 11.0 software. The results were presented as the mean \pm SEM. The sources of variation were analyzed by the unpaired Student's t-test. A P-value of $<0.05$ was considered to denote statistical significance.

\section{Results}

Histological examination. Routine histological examination revealed little morphologic change in the rat hearts from each group (data not shown).

Immunohistochemical staining. Immunohistochemical staining was performed on formalin-fixed, paraffin-embedded sections from all parts of the heart including the conduction system.

Expression of $\alpha$-enolase protein. Positive staining for the $\alpha$-enolase antibody was detected mainly in the extracellular matrix, or was manifested as fine brown cytoplasmic granularity or surface membrane expression. Two observers examined the sections independently.

Positive expression of $\alpha$-enolase in the control (Fig. 1A) and GM-injured (Fig. 1B) rat hearts was noted. Total IOD expression of $\alpha$-enolase in the rat hearts from each group is shown in Table I. Total IOD expression of $\alpha$-enolase in hearts from rats after GM-induced toxicity was significantly higher than that in the control (Table I) $(\mathrm{P}<0.05)$.

Expression of caveolin protein. The caveolin staining was detected mainly in the extracellular matrix, or was manifested as fine brown cytoplasmic granularity or surface membrane expression.

The distribution of caveolin in the rat hearts of the control (Fig. 2A) and GM (Fig. 2B) groups is shown. Total caveolin IOD in the heart of animals subjected to GM-induced toxicity was significantly higher than that of the control hearts (Table I) $(\mathrm{P}<0.05)$.

\section{Discussion}

Although therapy with GM is widely used for the treatment of severe gram-negative bacterial infections, the undesirable side effects associated with it markedly decrease the quality of life of patients.

$\alpha$-enolase, also called non-neuronal enolase, belongs to a family of cytoplasmic and glycolytic enzymes (21). It is a glycolytic enzyme that also acts as a surface plasminogen receptor (22). $\alpha$-enolase plays a functional role in several physiological processes depending on the cellular localization (23). It is a metabolic enzyme involved in the synthesis of pyruvate (24), and it has been suggested that increased $\alpha$-enolase may contribute to acceleration of glycolysis in the preterm heart (25).

$\alpha$-enolase plays an important role in pathophysiological processes (26). It has been reported that $\alpha$-enolase is an important target for oxidative and nitrative modifications in diabetic cardiac proteins (27), and tyrosine nitration of $\alpha$-enolase may contribute to impaired glycolytic activity in diabetic cardiomyopathy (27). It was found that $\alpha$-enolase was dramatically upregulated in hypertrophic hearts (28). $\alpha$-enolase is known to be bound to neutrophils and interacts in the immune response, and thus may play a role in inflammation associated with human labour (29). It is also recognized as a virulence factor of various pathogenic bacteria facilitating plasminogen activation and host cell invasion (30).

Apart from its enzymatic function in the glycolytic pathway, $\alpha$-enolase has been implicated in numerous diseases, 

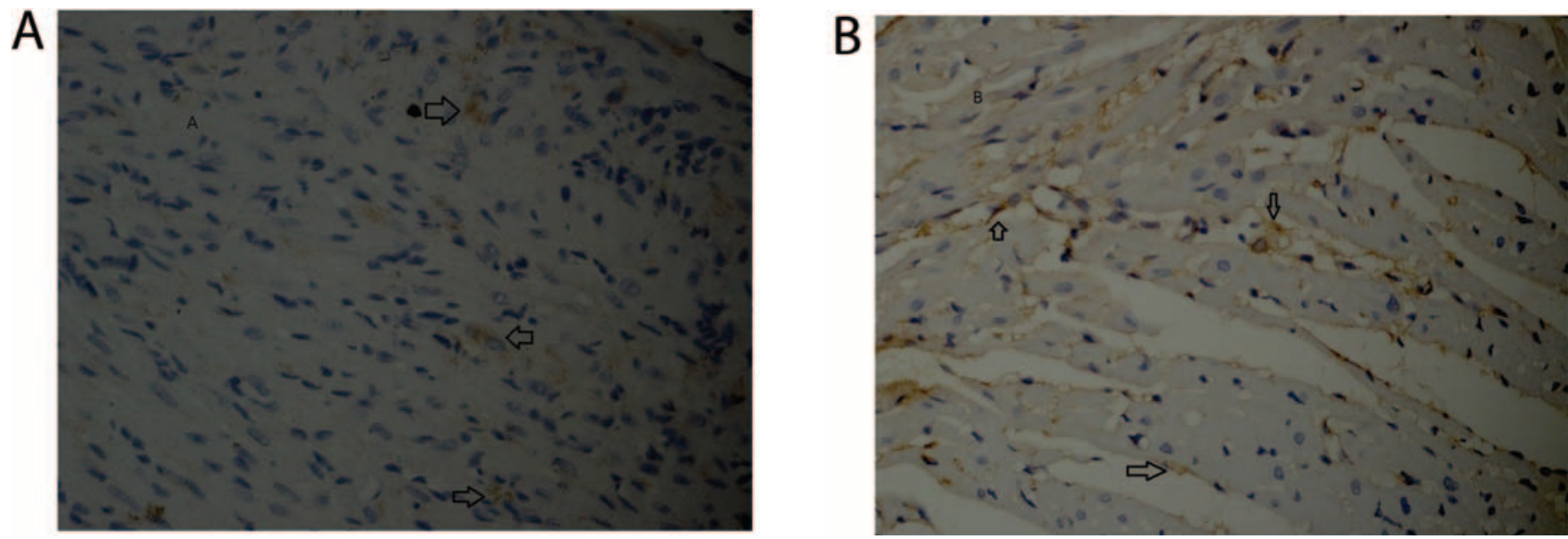

Figure 1. Effect of gentamicin-induced toxicity on $\alpha$-enolase expression in rat heart. Photomicrographs display a representative distribution of $\alpha$-enolasepositive expression in hearts from the (A) control and (B) gentamicin-induced group. All photomicrographs were captured at a magnification of x400. Positive immunostaining appears as brown staining (arrows).
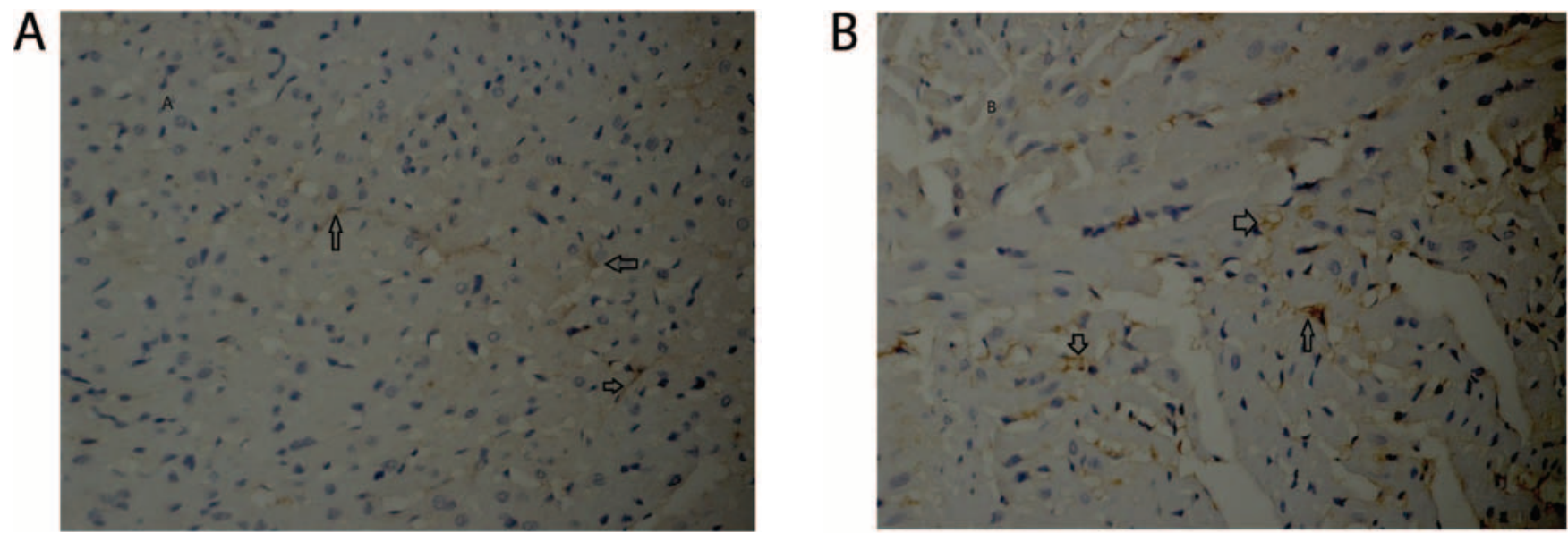

Figure 2. Effect of gentamicin-induced toxicity on caveolin expression in rat hearts. Photomicrographs reveal a representative distribution of positive caveolin expression in hearts from the (A) control and (B) gentamicin-induced toxicity group. All photomicrographs were captured at a magnification of x400. Positive immunostaining appears as brown staining (arrows).

Table I. The integrated optical density (IOD) of $\alpha$-enolase and caveolin in heart of rats.

\begin{tabular}{lcc}
\hline Groups & $\alpha$-enolase & Caveolin \\
\hline Control group & $0.0071 \pm 0.00035$ & $0.0077 \pm 0.00064$ \\
Gentamicin group & $0.0149 \pm 0.00027$ & $0.0189 \pm 0.00078$ \\
\hline
\end{tabular}

The integrated optical density (IOD) per field is proportional to the total amount of staining. Total $\alpha$-enolase IOD expression in rat hearts having undergone gentamicin-induced toxicity was significantly higher compared with control hearts $(\mathrm{P}<0.05)$. Total caveolin IOD expression in rat hearts subjected to gentamicin-induced toxicity was significantly higher compared with the control $(\mathrm{P}<0.05)$.

including metastatic cancer, autoimmune disorders, ischemia and bacterial infection (31). $\alpha$-enolase is a key glycolytic enzyme that has been used as a diagnostic marker to identify human lung cancers (32). Increased $\alpha$-enolase in the heart noted in our results suggests that the tissues were extensively damaged when undergoing GM-induced toxicity. The present study indicates that GM-induced toxicity causes increased $\alpha$-enolase expression that is related to impaired heart function.

Caveolin, a member of the membrane-anchoring protein family, accumulates various growth receptors in caveolae and function in a variety of biological processes (33). It has been reported that caveolae are organelles abundant in the plasma membrane of many specialized cells including endothelial cells, epithelial cells and adipocytes (34). Previous research has demonstrated that caveolae are small, flask-like invaginations of the plasma membrane, and caveolin are structural proteins found in caveolae that have scaffolding properties to allow the organization of signaling (35). Caveolin and caveolae have been implicated as being involved in the signal transduction of many receptors, including the EGF, PDGF, LPA and $\beta 3$-adrenergic receptors (36). It has been suggested that the ATP-sensitive $\mathrm{K}^{+}$channels in the heart are localized in caveolae (37). Caveolins consist of three different membrane 
scaffolding proteins that play a role in a variety of processes in different tissues (38). It has been reported that upregulation of caveolin attenuates cellular proliferation and growth (33). It has also been shown that the caveolins are the major structural proteins of caveolae and play a role in tumor surveillance (39). The increased levels of caveolin in the heart noted in our results suggests that the tissues were greatly damaged when undergoing GM-induced toxicity. The present study revealed that GM-induced toxicity caused increased caveolin expression related to impaired heart function.

Our results demonstrated that cardiac protein expression of $\alpha$-enolase and caveolin is altered after GM-induced toxicity in the rat. After undergoing GM-induced toxicity, the protein expression of $\alpha$-enolase and caveolin was significantly elevated. The present study indicated that GM-induced toxicity caused different alterations in heart proteins that may be related to cardiac-function deterioration.

In conclusion, the current findings indicate that such alterations in the protein expression of $\alpha$-enolase and caveolin would be reflected in abnormal cardiac function. These proteins may be useful in elucidating the mechanisms underlying GM-induced toxicity and may also provide certain clues for further research.

\section{Acknowledgements}

This study was supported by a grant from the Natural Science Foundation of Guangdong Province, China (4203003) and Guangdong Medical College (no. XQ0426).

\section{References}

1. Abdel-Raheem IT,El-Sherbiny GA and Taye A: Green tea ameliorates renal oxidative damage induced by gentamicin in rats. Pak J Pharm Sci 23: 21-28, 2010.

2. Ozbek E, Cekmen M, Ilbey YO, Simsek A, Polat EC and Somay A: Atorvastatin prevents gentamicin-induced renal damage in rats through the inhibition of p38-MAPK and NF-kappaB pathways. Ren Fail 31: 382-392, 2009.

3. Nishimura B, Tabuchi K, Nakamagoe M and Hara A: The influences of sphingolipid metabolites on gentamicin-induced hair cell loss of the rat cochlea. Neurosci Lett 485: 1-5, 2010.

4. Yang TH, Young YH and Liu SH: EGb 761 (Ginkgo biloba) protects cochlear hair cells against ototoxicity induced by gentamicin via reducing reactive oxygen species and nitric oxide-related apoptosis. J Nutr Biochem 22: 886-894, 2011.

5. Pessoa EA, Convento MB, Silva RG, Oliveira AS, Borges FT and Schor N: Gentamicin-induced preconditioning of proximal tubular LLC-PK1 cells stimulates nitric oxide production but not the synthesis of heat shock protein. Braz J Med Biol Res 42: 614-620, 2009

6. Abdel-Naim AB, Abdel-Wahab MH and Attia FF: Protective effects of vitamin e and probucol against gentamicin-induced nephrotoxicity in rats. Pharmacol Res 40: 183-187, 1999.

7. Abdel-Raheem IT, Abdel-Ghany AA and Mohamed GA: Protective effect of quercetin against gentamicin-induced nephrotoxicity in rats. Biol Pharm Bull 32: 61-67, 2009.

8. Said MM: The protective effect of eugenol against gentamicininduced nephrotoxicity and oxidative damage in rat kidney. Fundam Clin Pharmacol 25: 708-716, 2010.

9. Jeong SW, Kim LS, Hur D, Bae WY, Kim JR and Lee JH: Gentamicin-induced spiral ganglion cell death: apoptosis mediated by ROS and the JNK signaling pathway. Acta Otolaryngol 130: 670-678, 2010.

10. Petronilho F, Constantino L, de Souza B, et al: Efficacy of the combination of $\mathrm{N}$-acetylcysteine and desferrioxamine in the prevention and treatment of gentamicin-induced acute renal failure in male Wistar rats. Nephrol Dial Transplant 24: 2077-2082, 2009.
11. Al-Shabanah OA, Aleisa AM, Al-Yahya AA, et al: Increased urinary losses of carnitine and decreased intramitochondrial coenzyme A in gentamicin-induced acute renal failure in rats. Nephrol Dial Transplant 25: 69-76, 2010.

12. Ozaki N, Matheis KA, Gamber M, et al: Identification of genes involved in gentamicin-induced nephrotoxicity in rats - a toxicogenomic investigation. Exp Toxicol Pathol 62: 555-566, 2010.

13. Romero F, Perez M, Chavez M, Parra G and Durante P: Effect of uric acid on gentamicin-induced nephrotoxicity in rats - role of matrix metalloproteinases 2 and 9. Basic Clin Pharmacol Toxicol 105: 416-424, 2009.

14. Morales AI, Detaille D, Prieto M, et al: Metformin prevents experimental gentamicin-induced nephropathy by a mitochondria-dependent pathway. Kidney Int 77: 861-869, 2010.

15. Ajami M, Eghtesadi S, Pazoki-Toroudi H, Habibey R and Ebrahimi SA: Effect of Crocus sativus on gentamicin-induced nephrotoxicity. Biol Res 43: 83-90, 2010.

16. Helin HO, Lundin ME, Laakso M, Lundin J, Helin HJ and Isola J: Virtual microscopy in prostate histopathology: simultaneous viewing of biopsies stained sequentially with hematoxylin and eosin, and alpha-methylacyl-coenzyme A racemase/p63 immunohistochemistry. J Urol 175: 495-499, 2006.

17. De Rossi A, Rocha LB and Rossi MA: Application of fluorescence microscopy on hematoxylin and eosin-stained sections of healthy and diseased teeth and supporting structures. J Oral Pathol Med 36: 377-381, 2007.

18. Smith PS, Parkinson IH and Leong AS: Principles of ploidy analysis by static cytometry. Clin Mol Pathol 49: M104-M111, 1996.

19. Dong J, Yin H, Liu W, Wang P, Jiang Y and Chen J: Congenital iodine deficiency and hypothyroidism impair LTP and decrease $\mathrm{C}$-fos and C-jun expression in rat hippocampus. Neurotoxicology 26: 417-426, 2005.

20. van Kuijk AW, Gerlag DM, Vos K, et al: A prospective, randomised, placebo-controlled study to identify biomarkers associated with active treatment in psoriatic arthritis: effects of adalimumab treatment on synovial tissue. Ann Rheum Dis 68: 1303-1309, 2009.

21. Terrier B, Degand N, Guilpain P, Servettaz A, Guillevin L and Mouthon L: Alpha-enolase: a target of antibodies in infectious and autoimmune diseases. Autoimmun Rev 6: 176-182, 2007.

22. Cappello P, Tomaino B, Chiarle R, et al: An integrated humoral and cellular response is elicited in pancreatic cancer by alpha-enolase, a novel pancreatic ductal adenocarcinoma-associated antigen. Int J Cancer 125: 639-648, 2009.

23. Perconti G, Ferro A, Amato F, et al: The kelch protein NS1-BP interacts with alpha-enolase/MBP-1 and is involved in c-Myc gene transcriptional control. Biochim Biophys Acta 1773: 1774-1785, 2007.

24. Capello M, Ferri-Borgogno S, Cappello P and Novelli F: $\alpha$-Enolase: a promising therapeutic and diagnostic tumor target. FEBS J 287: 1064-1074, 2011.

25. Tsuzuki Y, Takeba Y, Kumai T, et al: Antenatal glucocorticoid therapy increase cardiac alpha-enolase levels in fetus and neonate rats. Life Sci 85: 609-616, 2009.

26. Chun JK, Lee TJ, Choi KM, Lee KH and Kim DS: Elevated anti-alpha-enolase antibody levels in Kawasaki disease. Scand J Rheumatol 37: 48-52, 2008

27. Lu N, Zhang Y, Li H and Gao Z: Oxidative and nitrative modifications of alpha-enolase in cardiac proteins from diabetic rats. Free Radic Biol Med 48: 873-881, 2010.

28. Zhu LA, Fang NY, Gao PJ, Jin X and Wang HY: Differential expression of alpha-enolase in the normal and pathological cardiac growth. Exp Mol Pathol 87: 27-31, 2009.

29. Dellios NL, Lappas M, Young IR, et al: Increased expression of alpha-enolase in cervico-vaginal fluid during labour. Eur J Obstet Gynecol Reprod Biol 153: 16-22, 2010.

30. Chumchua V, Pornputtapong N, Thammarongtham $\mathrm{C}$ and Meksuriyen D: Homology modeling of Mycoplasma pneumoniae enolase and its molecular interaction with human plasminogen. Bioinformation 3: 18-23, 2008.

31. Kang HJ, Jung SK, Kim SJ and Chung SJ: Structure of human alpha-enolase (hENO1), a multifunctional glycolytic enzyme. Acta Crystallogr D Biol Crystallogr 64: 651-657, 2008.

32. Tu SH, Chang CC, Chen CS, et al: Increased expression of enolase alpha in human breast cancer confers tamoxifen resistance in human breast cancer cells. Breast Cancer Res Treat 121: 539-553, 2010 . 
33. Otsu K, Toya Y, Oshikawa J, et al: Caveolin gene transfer improves glucose metabolism in diabetic mice. Am J Physiol Cell Physiol 298: C450-C456, 2010.

34. Davalos A, Fernandez-Hernando C, Sowa G, et al: Quantitative proteomics of caveolin-1-regulated proteins: characterization of polymerase $\mathrm{i}$ and transcript release factor/CAVIN-1 IN endothelial cells. Mol Cell Proteomics 9: 2109-2124, 2010.

35. Tsutsumi YM, Kawaraguchi Y, Horikawa YT, et al: Role of caveolin-3 and glucose transporter- 4 in isoflurane-induced delayed cardiac protection. Anesthesiology 112: 1136-1145, 2010

36. Mattsson CL, Andersson ER and Nedergaard J: Differential involvement of caveolin-1 in brown adipocyte signaling: impaired beta3-adrenergic, but unaffected LPA, PDGF and EGF receptor signaling. Biochim Biophys Acta 1803: 983-989, 2010.
37. Sun $\mathrm{W}$ and $\mathrm{Hu} \mathrm{K}$ : Role for SUR2A in coupling cardiac K(ATP) channels to caveolin-3. Cell Physiol Biochem 25: 409-418, 2010.

38. Rossi S, Poliani PL, Cominelli M, et al: Caveolin 1 is a marker of poor differentiation in rhabdomyosarcoma. Eur J Cancer 47: 761-772, 2010.

39. Amorim I, Lopes CC, Faustino AM and Pereira PD: Immunohistochemical expression of caveolin-1 in normal and neoplastic canine mammary tissue. J Comp Pathol 143: 39-44, 2010. 